\title{
Expression of Snail and Slug in renal cell carcinoma: E-cadherin repressor Snail is associated with cancer invasion and prognosis
}

\author{
Shuji Mikami ${ }^{1}$, Ken-Ichi Katsube ${ }^{2}$, Mototsugu Oya ${ }^{3}$, Masaru Ishida ${ }^{3}$, Takeo Kosaka ${ }^{3}$, Ryuichi Mizuno ${ }^{3}$, Makio Mukai ${ }^{1}$ \\ and Yasunori Okada ${ }^{4}$
}

The Snail family transcription factors have been proposed as important mediators of epithelial-mesenchymal transition because of their role in down-regulation of E-cadherin and up-regulation of matrix metalloproteinases (MMPs).

The present study was undertaken to investigate the expression of Snail, Slug and their associations with cancer invasion and prognosis in renal cell carcinomas (RCCs). Ninety-seven primary RCCs were analyzed for the protein expression of Snail, Slug, MMP2 and MMP9 by immunohistochemistry. Snail protein expression level was positively correlated with pathological tumor stage, histological grade and the presence of sarcomatoid carcinoma. On the contrary, Slug protein expression level was negatively correlated with pathological tumor stage, suggesting that Slug was down-regulated in advanced RCCs. Because Snail was positively associated with malignant potential of RCCs, involvement of Snail in the invasiveness of an RCC cell line 786-O was examined in the Matrigel invasion assay by down-regulating the gene expression with small interfering RNA (siRNA). Targeting the Snail, not Slug, expression in 786-O cells with siRNA caused down-regulation of the gene expression of Snail, vimentin, MMP2 and MMP9, but up-regulated the E-cadherin. Invasion of the cells through Matrigel in vitro was inhibited under this condition. Furthermore, expression levels of MMP2 and MMP9 were positively correlated with pathological tumor stage and the presence of sarcomatoid carcinoma. Statistical analysis indicated that elevated Snail, MMP2 and MMP9 protein expression are significantly worse predictors of diseasefree and disease-specific survival of the patients with RCC. In conclusion, these data suggest that Snail has an important role in invasion and metastasis, and that silencing the gene may be a potential therapeutic target in RCCs.

Laboratory Investigation (2011) 91, 1443-1458; doi:10.1038/labinvest.2011.111; published online 1 August 2011

KEYWORDS: E-cadherin; epithelial-mesenchymal transition; matrix metalloproteinase; renal cell carcinoma; Slug; Snail

Renal cell carcinoma (RCC) is the most common malignancy of the kidney, ${ }^{1}$ and $20-30 \%$ of patients who undergo curative surgery will develop metastatic disease during follow-up. ${ }^{2}$ Tumor metastasis depends on the ability of cancer cells to invade tissue barriers composed of basement membrane and extracellular matrix. ${ }^{3}$ Matrix metalloproteinases (MMPs) may have important roles in tumorigenesis and cancer cell progression because they digest the main components of basement membrane and extracellular matrix. ${ }^{4}$ In fact, Kugler et al. ${ }^{5}$ reported a strong correlation between increased expression of MMP2 and MMP9 and tumor stage in RCCs.

Epithelial-mesenchymal transition (EMT), a switch of polarized epithelial cells to a migratory, sarcomatoid pheno- type, is considered to be an important event during malignant tumor progression and metastasis. ${ }^{6}$ A hallmark of EMT is the loss of cell adhesion molecule E-cadherin, and several EMT regulators have been identified as E-cadherin repressors. ${ }^{7}$ Among them, Snail and Slug appear to be key regulators of EMT as they repress the transcription of E-cadherin, thereby triggering a complete EMT with an acquisition of invasive and tumorigenic properties. ${ }^{8,9}$ Snail expression has been detected in many malignant tumors and is associated with invasiveness and metastatic potential of the tumors, suggesting that Snail is a key molecule in the induction of tumor invasion and metastasis. ${ }^{8,9}$ It has been reported that loss of E-cadherin expression is associated with

\footnotetext{
${ }^{1}$ Division of Diagnostic Pathology, Keio University Hospital, Tokyo, Japan; ${ }^{2}$ Department of Molecular Pathology, Graduate School of Tokyo Medical and Dental University, Tokyo, Japan; ${ }^{3}$ Department of Urology, School of Medicine, Keio University, Tokyo, Japan and ${ }^{4}$ Department of Pathology, School of Medicine, Keio University, Tokyo, Japan

Correspondence: Dr S Mikami, MD, PhD, Division of Diagnostic Pathology, Keio University Hospital, 35 Shinanomachi, Shinjuku-ku, Tokyo 160-8582, Japan.

E-mail: mikami@a7.keio.jp

Received 11 August 2010; revised 23 May 2011; accepted 2 June 2011
} 
metastasis of RCC, ${ }^{10}$ and in vitro studies suggested the possibility that E-cadherin repressor Snail may be involved in EMT of RCC. ${ }^{11-13}$ However, to the best of our knowledge, there has been no report on the role of Snail and Slug in EMT of RCC in vivo.

Here, we show that Snail is expressed in RCCs with positive associations with primary tumor stage and nuclear grade. In contrast, Slug expression is negatively associated with primary tumor stage, suggesting the down-regulation of Slug expression during malignant progression of RCCs. In addition, down-regulation of Snail by treatment of an RCC cell line, 786-O, with small interfering RNA (siRNA) led to down-regulation of MMP2 and MMP9, and up-regulation of E-cadherin together with inhibition of the cell invasion through Matrigel in vitro, whereas siRNA for Slug showed no such effects. These results can open the way for therapeutic approaches based on the use of Snail-specific siRNA as new cancer strategies.

\section{MATERIALS AND METHODS Patients}

Ninety-seven patients with RCC (83 clear cell, 10 papillary and 4 chromophobe RCC) were included in the present study. Small pieces of clear cell RCCs $(n=25)$ and control non-neoplastic renal tissues $(n=7)$ were frozen for real-time polymerase chain reaction (PCR) analysis. As for seven clear cell RCCs (four low-grade and three high-grade tumors), tumor tissues were available for immunoblot analysis. Seventy-four patients were males and 23 patients were females. Their clinicopathological characters were summarized in Table 1. During follow-up, 11 patients developed metastastic disease and 9 patients died of the disease. Specimens of seven metastastic RCCs (three bone metastases, two liver metastases, one skin metastasis and one lung metastasis) were available for histological analysis. Informed consent for experimental use of the samples was obtained from the patients according to the hospital's ethical guidelines.

\section{Polymerase Chain Reaction}

RNA isolation, cDNA synthesis and quantitative PCR were performed as described previously. ${ }^{14}$ The primers and TaqMan probes sets (TaqMan Gene Expression Assays, Inventoried) for Snail (Hs00195591_m1), Slug (Hs00950344_m1), E-cadherin (Hs001170423_m1), vimentin (Hs00185584_m1), MMP2 (Hs00234422_m1), MMP9 (Hs00234579_ml), MMP14 (Hs00237119_ml) and human $\beta$-actin endogenous control (4310881E) were purchased from Applied Biosystems (sequences not disclosed). Ratios of Snail, Slug, E-cadherin, vimentin and MMPs to $\beta$-actin mRNA were calculated for each sample to evaluate the relative mRNA expression.

\section{Immunoblotting}

Protein samples were analyzed by sodium dodecyl sulfatepolyacrylamide gel electrophoresis (10\% total acrylamide) under reduction followed by transfer to polyvinylidene difluoride membranes. Antibodies were used at the following concentrations: rabbit polyclonal anti-Snail antibodies (Abcam, Cambridge, MA, USA), $1 \mu \mathrm{g} / \mathrm{ml}$; rabbit polyclonal anti-Slug antibodies (Abcam), $1 \mu \mathrm{g} / \mathrm{ml}$; anti-E-cadherin mouse monoclonal antibody (clone: 36/E-cadherin; BD Biosciences, San Jose, CA, USA), $0.5 \mu \mathrm{g} / \mathrm{ml}$; mouse monoclonal anti- $\beta$-actin antibodies (Sigma, St Louis, MO, USA), $1 \mu \mathrm{g} / \mathrm{ml}$. The immunoreactive protein bands were detected with enhanced chemiluminescence immunoblotting western blotting reagents (Amersham, Piscataway, NJ, USA).

\section{Immunohistochemistry}

Paraffin sections were reacted with rabbit polyclonal antiSnail antibodies $(2 \mu \mathrm{g} / \mathrm{ml}$; Abcam $)$, rabbit polyclonal antiSlug antibodies $(2 \mu \mathrm{g} / \mathrm{ml}$; Abcam), anti-E-cadherin mouse monoclonal antibody (clone: 36/E-cadherin, $1.25 \mu \mathrm{g} / \mathrm{ml}$; BD Biosciences), rabbit polyclonal anti-MMP2 antibodies $(0.2 \mu \mathrm{g} / \mathrm{ml}$; Abnova, Taipei, Taiwan) or rabbit polyclonal anti-MMP9 antibodies $(2 \mu \mathrm{g} / \mathrm{ml}$; Abnova) as described previously. ${ }^{14}$ For negative controls, the primary antibodies were replaced with non-immune rabbit $(2 \mu \mathrm{g} / \mathrm{ml})$ or mouse immunoglobulin $\mathrm{G}(\mathrm{IgG})(1.25 \mu \mathrm{g} / \mathrm{ml})$.

To evaluate Snail and Slug staining, carcinoma cells with positive nuclear staining were counted in at least 10 representative fields ( $\times 400$ magnification), and the mean and median percentage of positive cancer cells was calculated. The cases in which the positive tumor cell ratio was equal to or more than the median were defined as high cases, and those less than the median were defined as low cases. To evaluate E-cadherin staining, carcinoma cells with positive cell surface staining were counted, and the mean percentage of positive cells was calculated. To evaluate MMP2 and MMP9 staining, carcinoma cells with positive cell surface and/or cytoplasmic staining were counted, and the mean and median percentage of positive cells was calculated. E-cadherin, MMP2 or MMP9-high and low cases were defined as described above according to the classification of Snail and Slug-positive cases.

\section{Cell Culture and siRNA}

786-O and ACHN cells, human clear cell RCC cell lines (American Type Culture Collection, Manassas, VA, USA) were cultured in RPMI1640 medium supplemented with $10 \%$ fetal bovine serum (FBS), penicillin and streptomycin (Sigma-Aldrich, St Louis, MO, USA). Pre-designed siRNAs for Snail, siSnail\#1 and siSnail\#2 (S13185 and S13186; Silencer ${ }^{\circledR}$ Select Pre-designed siRNA), and siRNAs for Slug, siSlug\#1 and siSlug\#2 (S13127 and S13128), were purchased from Ambion (Austin, TX, USA). Sequences of siSnail\#1, siSnail\#2, siSlug\#1 and siSlug\#2 were as follows: GGACA AAGGCUGACAGACUTT; GAAUGUCCCUGCUCCACAA TT, CAAUAAGACCUAUUCAACUTT, GCAGACAGGUCAA AUCUGATT, respectively. Non-silencing siRNA (NSi; AllStars Negative Control siRNA) was obtained from Qiagen 
Table 1 Relationship between expression of Snail, Slug, E-cadherin and clinicopathological parameters in renal cell carcinomas

Snail-positive tumor

cells (\%) median

(25th-75th percentile)
Slug-positive tumor

cells (\%) median

(25th-75th percentile)
E-cadherin-positive tumor cells (\%) median (25th-75th percentile)

Gender

Male $(n=74)$

Female $(n=23)$

$P$

Age (years)

Median or less $(\leq 59)(n=48)$

Over median $(>59)(n=49)$

$P$

\section{pT1, $2(n=78)$ \\ pT3, $4(n=19)$ \\ $P$}

Pathological tumor stage

Lymph node metastasis

Negative $(n=93)$

Positive $(n=4)$

$P$

\section{Distant metastasis}
pM0 $(n=92)$
pM1 $(n=5)$
P
$22.5(1.5-51.5)$
$38.0(24.0-74.5)$
0.278

Histological grade
G1, $2(n=74)$
$\mathrm{G} 3,4(n=23)$
$P$

Venous invasion

$$
\begin{aligned}
& \text { Negative }(n=74) \\
& \text { Positive }(n=23)
\end{aligned}
$$$$
P
$$

\section{Sarcomatoid carcinoma}

$$
\begin{aligned}
& \text { Negative }(n=90) \\
& \text { Positive }(n=7) \\
& P
\end{aligned}
$$

$$
\begin{gathered}
24.5(1.5-45.0) \\
23.0(2.3-67.0) \\
0.840
\end{gathered}
$$

$$
\begin{gathered}
19.0(0-37.0) \\
65.0(19.3-77.0) \\
0.010
\end{gathered}
$$

$$
\begin{gathered}
19.5(0-45.0) \\
71.0(50.5-83.0) \\
0.005
\end{gathered}
$$

$$
\begin{gathered}
8.0(0-30) \\
1.0(0-29.3) \\
0.084
\end{gathered}
$$$$
11.0(0-35.0)
$$$$
15.0(0-32.5)
$$$$
0.725
$$

$$
\begin{gathered}
5.0(0-34.0) \\
5.0(0-30.0) \\
0.974
\end{gathered}
$$$$
0(0-25.0)
$$$$
20.0(0-52.0)
$$$$
0.012
$$

$$
\begin{gathered}
10.0(0-33.0) \\
1.0(0-3.0) \\
0.010
\end{gathered}
$$$$
5.0(0-35.0)
$$$$
17.0(0-24.5)
$$$$
0.737
$$

$$
\begin{gathered}
5.0(0-30.8) \\
2.5(1.0-9.0) \\
0.469
\end{gathered}
$$$$
12.0(0-35.0)
$$$$
15.0(3.5-29.0)
$$$$
0.849
$$

$$
\begin{gathered}
5.5(0-31.5) \\
2.5(0.8-5.3) \\
0.336
\end{gathered}
$$$$
13.5(0-35.0)
$$$$
7.0(1.5-30.0)
$$$$
0.877
$$

$$
\begin{gathered}
9.5(0-33.0) \\
2.0(0-12.3) \\
0.124
\end{gathered}
$$$$
6.5(0-35.0)
$$$$
17.0(0-35.0)
$$$$
0.579
$$

$$
\begin{gathered}
6.5(0-33.0) \\
2.0(0-15.0) \\
0.197
\end{gathered}
$$$$
9.0(0-35.0)
$$$$
12.0(0-32.5)
$$$$
0.539
$$

$$
\begin{gathered}
6.5(0-30.0) \\
2.0(0-2.0) \\
0.1432
\end{gathered}
$$$$
13.5(0-35.0)
$$

KK (Tokyo, Japan). Cells were transfected with siSnail\#1, siSnail\#2 or NSi using Lipofectamine 2000 (Invitrogen, Tokyo, Japan) as described previously. ${ }^{14}$ After $24 \mathrm{~h}$, siRNAs were removed by changing the culture medium with fresh RPMI1640 containing 10\% FBS, and cells were cultured for additional $48 \mathrm{~h}$. There was no significant difference between 
the proliferation of cells transfected with NSi and those with siRNA for Snail and Slug (data not shown).

\section{Migration Assays}

Migration assays were performed by seeding $1 \times 10^{4}$ cells in $100 \mu \mathrm{l}$ of RPMI1640 on top of Transwell cell culture inserts consisting of a non-coated polyethylene terephthalate membrane (24-well inserts, $8.0 \mu \mathrm{m}$ pore size; Coster, Corning, Corning, NY, USA). The lower chamber was filled with $0.6 \mathrm{ml}$ of RPMI1640. After incubation for $24 \mathrm{~h}$, the non-migrating cells were scraped off, and the membranes were fixed and stained using the Diff-Quik ${ }^{\mathrm{TM}}$ stain kit (Sysmex, Hyogo, Japan). Cells that had migrated through the membranes were quantified by determination of the cell number in three randomly chosen visual fields at $\times 200$ magnification.

\section{Matrigel Invasion Assay}

Tumor cells in serum-free RPMI1640 were seeded on top of transwell inserts with $8 \mu \mathrm{m}$ pore-size polyethylene terephthalate membrane coated with Matrigel $^{\mathrm{TM}}$ basement membrane matrix (BD Biosciences), whereas the lower chamber was filled with RPMI1640 with 1\% FBS as chemoattractants. Cells were cultured for $24 \mathrm{~h}$ before the nonmigrating cells in the inserts were scraped off; membranes were fixed and stained using Diff-Quik ${ }^{\mathrm{TM}}$ stain kit (Sysmex). The cells that had migrated through the membrane were quantified by determination of the cell number in three randomly chosen visual fields at $\times 200$ magnification.

\section{Statistical Analysis}

Mann-Whitney's $U$-test was used to analyze the relationships between expression levels of Snail, Slug mRNA and E-cadherin mRNA, Snail, Slug, E-cadherin, MMPs protein positive tumor cell ratio and clinicopathological parameters. The logrank test and Kaplan-Meier method were used for survival analyses. Univariate and multivariate analyses were conducted according to Cox proportional hazard model. In vitro results were expressed as mean \pm s.d. Student's $t$-test was used for analyses related to in vitro experiments. $P$-values $<0.05$ were considered to be significant. StatView for Windows (version 5.0; Abacus Concepts, Berkeley, CA, USA) was used to calculate statistical differences between groups.

\section{RESULTS}

mRNA Expression of Snail, Slug and E-cadherin in RCCs and Non-Neoplastic Renal Tissues

Clear cell RCCs showed a slightly higher level of Snail mRNA (Snail mRNA/ $\beta$-actin mRNA) (mean \pm s.d.: $0.89 \pm 1.03$ ) than non-neoplastic renal tissues $(0.47 \pm 0.41)$, but the difference did not reach statistical significance $(P=0.309)$ (Figure 1a). Slug mRNA expression in clear cell RCCs $(1.89 \pm 1.86)$ was significantly lower than that in non-neoplastic renal tissues $(4.94 \pm 1.55) \quad(P<0.01) \quad$ (Figure $1 \mathrm{~b})$. The mean expression level of E-cadherin mRNA was significantly lower in clear cell
RCCs $(0.50 \pm 0.47)$ than in non-neoplastic renal tissues $(1.11 \pm 0.46)(P<0.01)$ (Figure 1c).

Correlations between the mRNA expression of, Snail, Slug and E-cadherin were analyzed. The tumors with high E-cadherin mRNA expression generally showed low Snail mRNA expression (Figure 1d), although there was no significant correlation between these factors $\left(R^{2}=0.08\right.$, $P=0.17)$. This was considered to be mainly due to the cases with low expression of both Snail and E-cadherin. There was no significant correlation between Slug and E-cadherin mRNA expression (Figure 1e; $R^{2}=0.02, P=0.53$ ), suggesting that Slug may not be a major E-cadherin repressor in clear cell RCC.

\section{Expression of Snail Protein in RCCs and Non-Neoplastic Renal Tissues}

Immunoblot analysis revealed that one of four low-grade clear cell RCCs showed weak Snail protein expression, and Snail protein expression was observed in all three high-grade RCCs (Figure 1f).

Snail immunostaining was observed in the nuclei of cancer cells in 70 of 83 clear cell RCCs (84\%), 3 of 10 papillary RCCs $(30 \%)$ and 0 of 4 chromophobe RCCs $(0 \%)$. In clear cell RCCs, cancer cells with G2 showed focal nuclear Snail staining (arrows in Figure 2a). Diffuse staining was observed in clear cell RCCs with G3 (Figure 2b). In papillary RCCs, cancer cells showed focal and weak nuclear staining (arrows in Figure 2c). No staining was observed in chromophobe RCC (Figure 2d). A sarcomatoid carcinoma component was observed in 7 of 83 clear cell RCCs $(8 \%)$, but not in 10 papillary RCCs $(0 \%)$ or 4 chromophobe RCCs $(0 \%)$, and all the cases of sarcomatoid carcinoma showed diffuse and strong Snail staining (Figure 2e). All seven metastastic clear cell RCCs showed diffuse and strong Snail expression (Figure 2f), and they showed higher Snail expression than their primary tumors. Snail staining was not observed in most non-neoplastic renal tissues (data not shown), but it was observed in stromal fibroblasts and inflammatory cells adjacent to cancer cells, which showed strong Snail staining (data now shown). However, in such cases, the renal tissues remote from cancer cells showed no apparent nuclear Snail staining.

\section{Expression of Slug Protein in RCCs and Non-Neoplastic Renal Tissues}

By immunoblot analysis, two low-grade clear cell RCCs showed Slug protein expression and two showed very weak expression (Figure 1f). All three high-grade clear cell RCCs showed very weak Slug protein expression.

Slug immunostaining was observed in the nuclei of cancer cells in 57 of 83 clear cell RCCs (69\%), 10 of 10 papillary RCCs (100\%) and 2 of 4 chromophobe RCCs (50\%). Clear cell RCCs with G2 showed diffuse nuclear Slug staining (Figure 2g). Only focal staining was observed in clear cell RCCs with G3 (Figure 2h), and all sarcomatoid carcinomas 

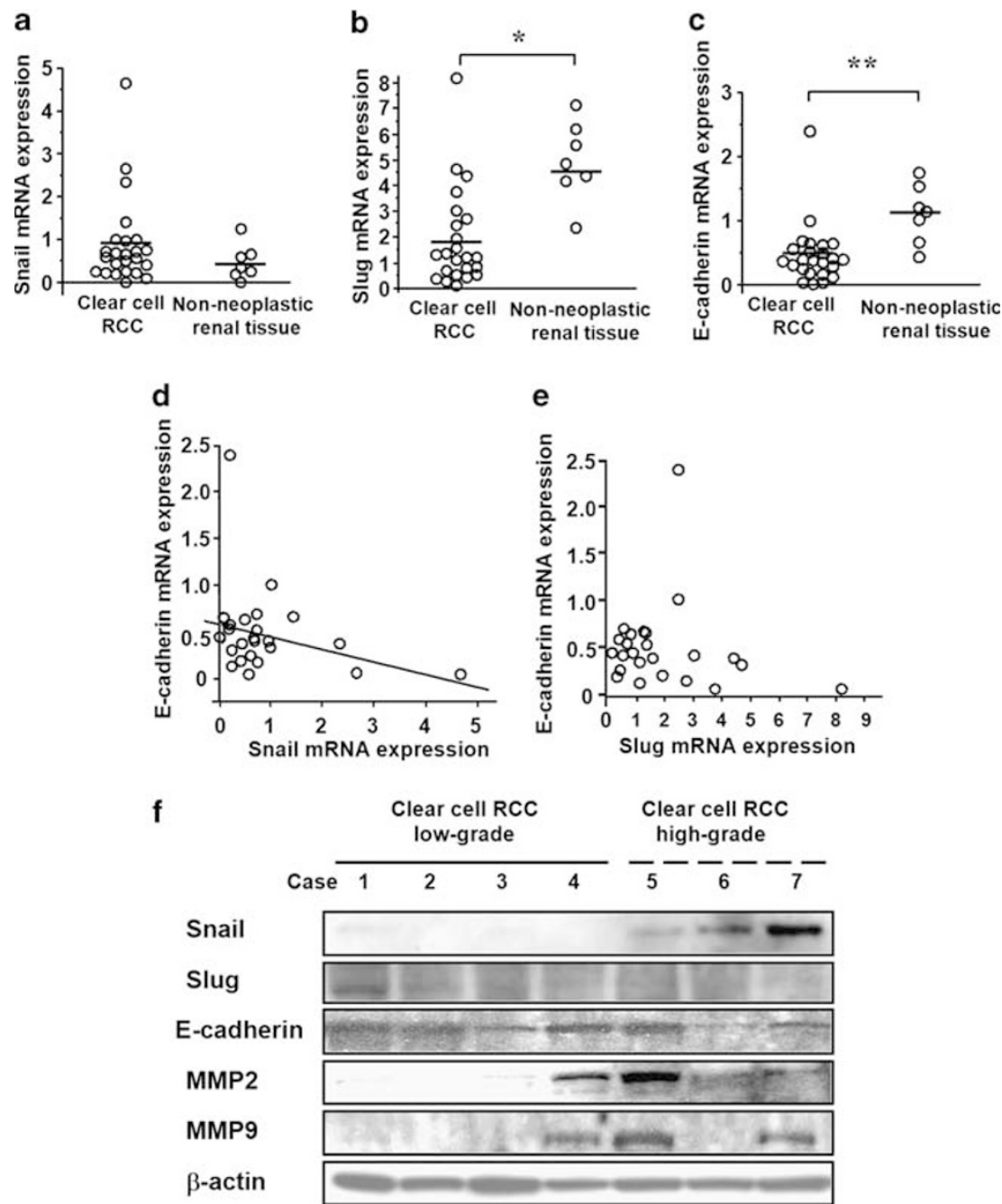

Figure 1 Relative mRNA expression of Snail and E-cadherin: Snail/ $\beta$-actin (a), Slug/ $\beta$-actin (b), E-cadherin/ $\beta$-actin (c) mRNA ratios were determined in primary RCCs and non-neoplastic renal tissues. Bars, mean values; ${ }^{\star} P<0.05 ;{ }^{*} P<0.01$. Correlations between Snail and E-cadherin mRNA expression (d) and Slug and E-cadherin expression (e). Immunoblot analysis of Snail, Slug, E-cadherin, MMP2 and MMP9 in four low-grade and three high-grade RCCs (f). Note that the patient having the tumor that showed strongest Snail protein expression (Case \#7 in $\mathbf{f}$ ) was pointed out a bone metastasis about 2 months after nephrectomy.

showed no Slug expression (Figure 2i). Focal Slug staining was observed in papillary and chromophobe RCC (data not shown). Nuclear Slug staining was also observed in the nuclei of renal tubules (data not shown).

\section{E-cadherin Protein Expression in RCCs and Non-Neoplastic Renal Tissues}

Three of four low-grade clear cell RCCs showed high E-cadherin protein expression, whereas only one of three highgrade tumors showed high E-cadherin expression (Figure 1f).

Cell surface E-cadherin staining was observed in 45 of 83 clear cell RCCs (54\%), 6 of 10 papillary RCCs (60\%) and 4 of 4 chromophobe RCCs (100\%). In clear cell RCCs with G2, many cancer cells showed cell surface E-cadherin staining (Figure 2j). In clear cell RCCs with G3, only focal cell surface staining was observed (Figure 2k). Many cancer cells of papillary RCCs showed cell surface E-cadherin staining (Figure 21). Diffuse and strong E-cadherin staining was observed in chromophobe RCC (Figure 2m). No cell surface E-cadherin staining was observed in the sarcomatoid carcinoma component (Figure 2n). Metastastic clear cell carcinoma showed no or markedly decreased cell surface E-cadherin expression (Figure 2o). All seven metastatic RCCs showed lower E-cadherin expression than their primary tumors. In non-neoplastic renal tissues, E-cadherin was expressed in distal and collecting tubules (data not shown).

\section{Associations Between Snail and Slug Immunohistochemical Expression, Histological Subtypes and Clinicopathological Parameters}

The Snail-positive tumor cell ratio varied from 0 to 99 , and its mean and median in all tumors (97 cases) including all 
subtypes of RCCs were 24 and 31, respectively. The ratio chromophobe RCCs $(0 \pm 0)(P<0.01)$ (Figure 3a). As shown in clear cell RCCs $(35 \pm 31$; mean \pm s.d. $)$ was significantly higher than that in papillary RCCs $(13 \pm 23)(P<0.05)$ or

in Table 1, Snail-positive cancer cell ratio was positively correlated with primary tumor stage $(P=0.010)$, histological

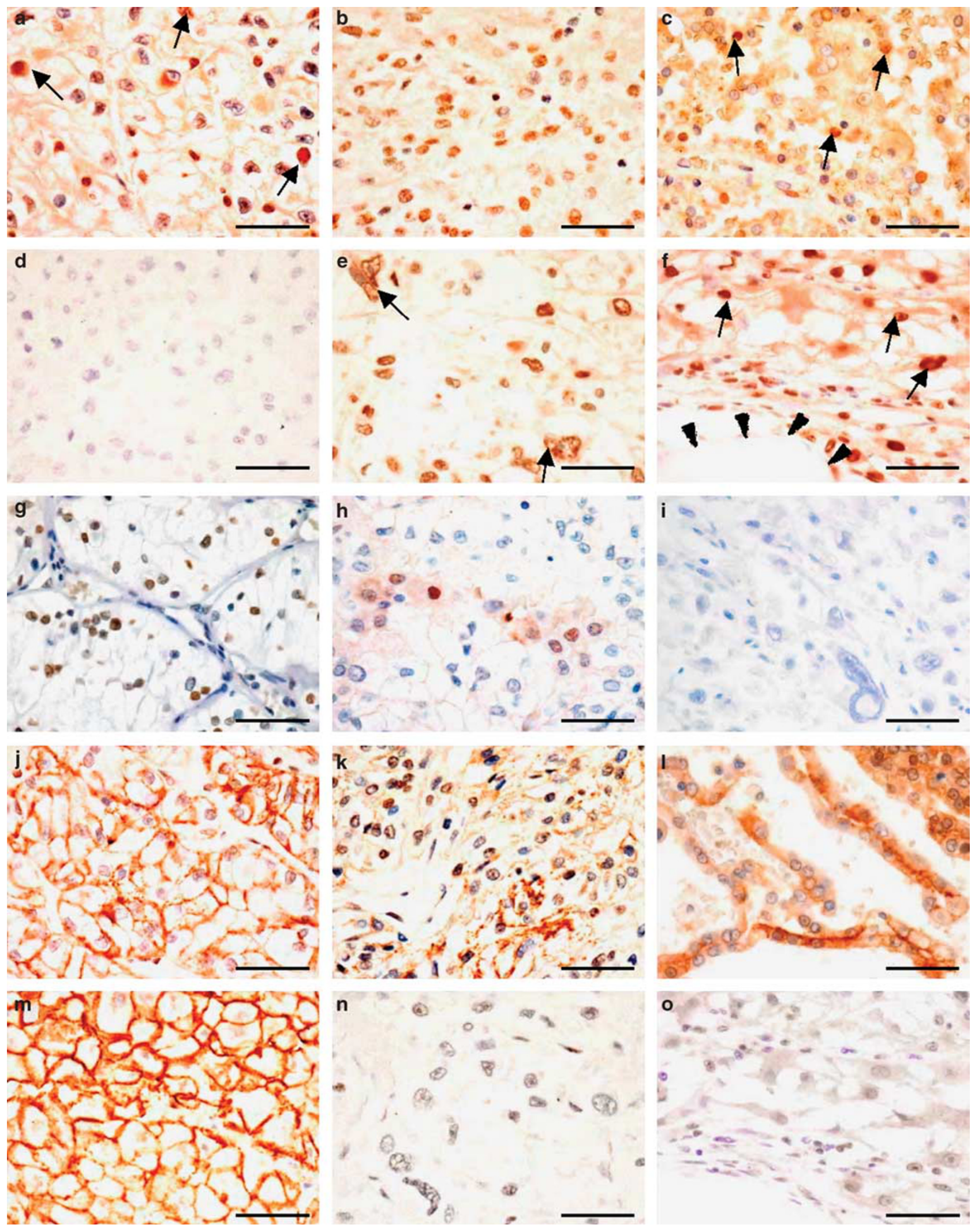


grade $(P=0.024)$ and the presence of sarcomatoid carcinoma $(P=0.005)$.

The Slug-positive tumor cell ratio varied from 0 to 98 , and its mean and median in all tumors (97 cases) including all subtypes of RCCs were 19 and 5, respectively. The ratio in clear cell RCCs $(17 \pm 24$; mean \pm s.d.) was significantly lower than that in papillary RCCs $(35 \pm 30)(P<0.05)$ (Figure 3b). No significant difference was observed between the Slugpositive tumor cell ratio in chromophobe RCCs (11 \pm 14$)$ and that in clear, or papillary RCCs. As shown in Table 1, Slug-positive cancer cell ratio was negatively correlated with primary tumor stage $(P=0.010)$.

\section{Associations Between E-cadherin Immunohistochemical Expression, Histological Subtypes and Clinicopathological Parameters}

The E-cadherin-positive tumor cell ratio varied from 0 to 100 , and its mean and median in all tumors (97 cases) including all subtypes of RCCs was 24 and 23. The mean E-cadherin-positive cancer cell ratio in chromophobe RCCs was $86 \pm 13$ and it was significantly higher than those in clear cell RCCs $(22 \pm 29)$ and papillary RCCs $(16 \pm 20)$ (Figure 3c). The E-cadherin-positive cancer cell ratio was higher in patients aged over 59 years than those aged 59 years or under it (Table $1 ; P=0.012$ ). There was no significant association
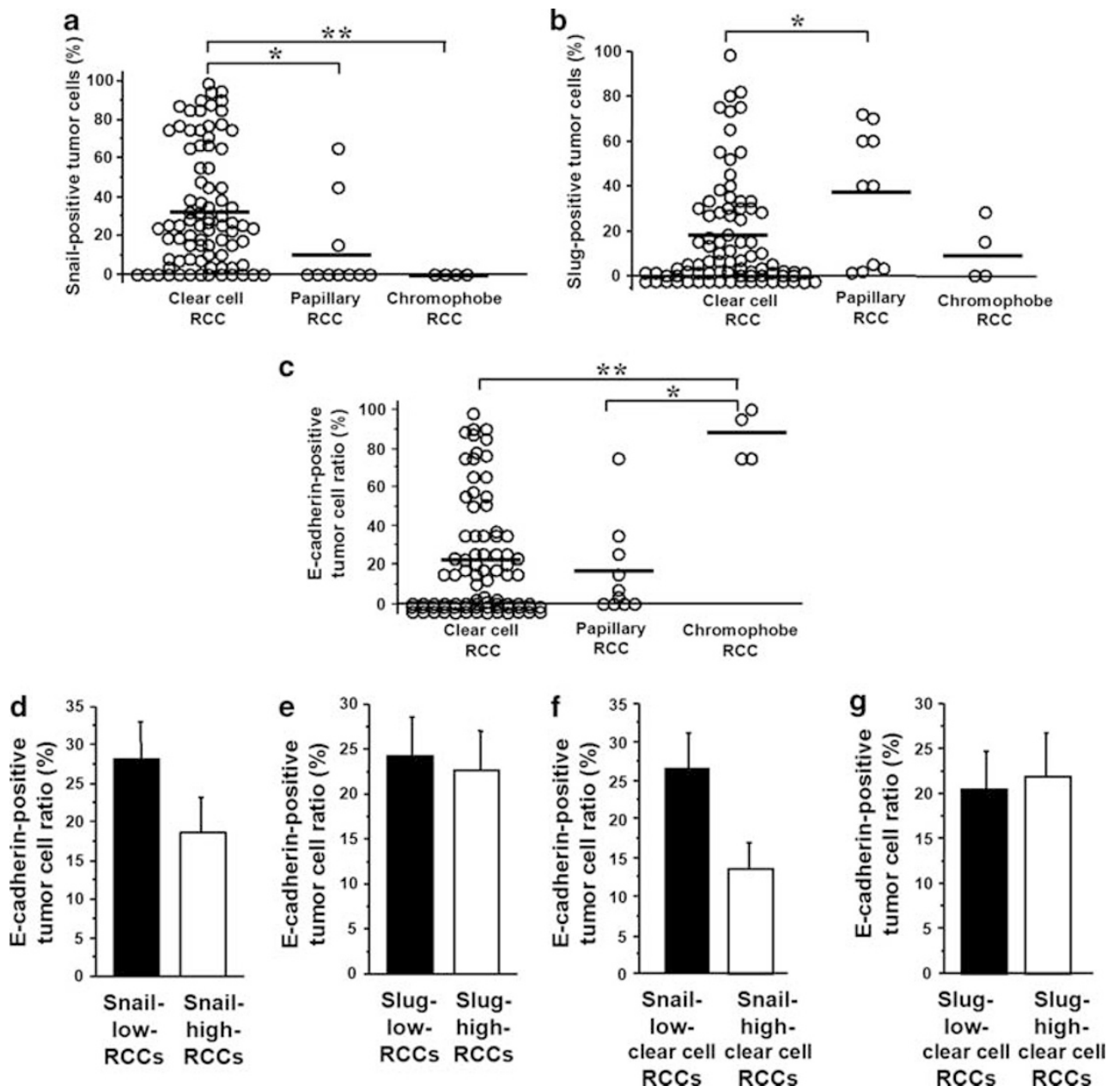

Figure 3 Correlations between Snail-positive tumor cell ratios and histological subtypes of RCCs (a), Slug (b) and E-cadherin-positive tumor cell ratio and histological subtypes of RCCs (c). Bars, mean; ${ }^{\star} P<0.05$; ${ }^{*} P<0.01$. Correlations between E-cadherin expression and Snail (d), or Slug (e) in all tumors (97 cases) including all subtypes of RCCs. Correlations between E-cadherin expression and Snail (f), or Slug (g) in 83 clear cell RCCs.

Columns, mean; bars, s.d.

Figure 2 Immunostaining of Snail in clear cell RCC, G2 (a), clear cell RCC, G3 (b), papillary RCC, G2 (c), chromophobe RCC (d), sarcomatoid carcinoma (e) and metastastic clear cell RCC in bone (f). Immunostaining of Slug in clear cell RCC, G2 (g), clear cell RCC, G3 (h) and sarcomatoid carcinoma (i).

Immunostaining of E-cadherin in clear cell RCC, G2 (j), clear cell RCC, G3 (k), papillary RCC, G2 (I), chromophobe RCC (m), sarcomatoid carcinoma (n) and metastastic clear cell RCC in bone (o). Bars, $50 \mu \mathrm{m}$. (a-j, b-k, c-l, d-m, e-n, $\mathbf{f}-\mathbf{o})$ Matched from the same patients. Note that some cancer cells in clear cell RCC, G2, showed Snail staining (arrows in a), while for papillary RCC, G2, only some cancer cells showed nuclear Snail staining (arrows in c); Strong Snail staining was observed in sarcomatoid carcinoma (arrows in e) and metastastic clear cell RCC in bone (f) (arrows, cancer cells; arrowheads, bone). 
between the E-cadherin-positive cancer cell ratio and other clinicopathological parameters.

\section{Association Between Snail, Slug and E-cadherin Immunohistochemical Expression in RCCs}

The immunohistochemical staining of cell surface E-cadherin staining was observed in RCCs with focal or no Snail staining (Figures $2 \mathrm{a}, \mathrm{d}, \mathrm{j}$ and $\mathrm{m}$ ). In contrast, it was markedly decreased or lost in RCCs with diffuse Snail staining (Figures $2 \mathrm{~b}, \mathrm{e}, \mathrm{f}, \mathrm{k}, \mathrm{n}$ and $\mathrm{o}$ ). In addition, the statistical analyses in 97 RCCs including all subtypes of RCCs showed that Snail-high RCCs showed lower E-cadherin-positive tumor cell ratio $(19 \pm 27)$ than that in Snail-low RCCs $(28 \pm 34)$, although the difference did not reach statistically significance $(P=0.127)$ (Figure 3d). In contrast, E-cadherin-positive tumor cell ratio in Slug-high RCCs was $23 \pm 32$, and it was similar to that in Slug-low RCCs $(24 \pm 30)(P=0.806)$ (Figure 3e).
When correlations between Snail, Slug and E-cadherin protein expression in 83 clear cell RCCs were analyzed, similar results were observed: Snail-low clear cell RCCs showed relatively high E-cadherin-positive tumor cell ratio compared with Snail-high tumors (Figure 3f), although the difference did not reach statistically significance $(P=0.19)$. There was no correlation between Slug and E-cadherin protein expression (Figure 3g; $P=0.77$ ), indicating that Slug is not involved in E-cadherin expression.

\section{Snail and Slug Expression in RCC Cell Lines and Their Migration and Invasion through Matrigel In Vitro}

Snail and Slug mRNA expression was higher in 786-O cells than in ACHN cells (Figures $4 \mathrm{a}$ and b). In contrast, E-cadherin expression was higher in ACHN cells than in 786-O cells (Figure 4c). Vimentin mRNA expression was lower in 786-O cells than in ACHN cells (Figure 4d). MMP2 and MMP9 mRNA expression was markedly higher in 786-O cells
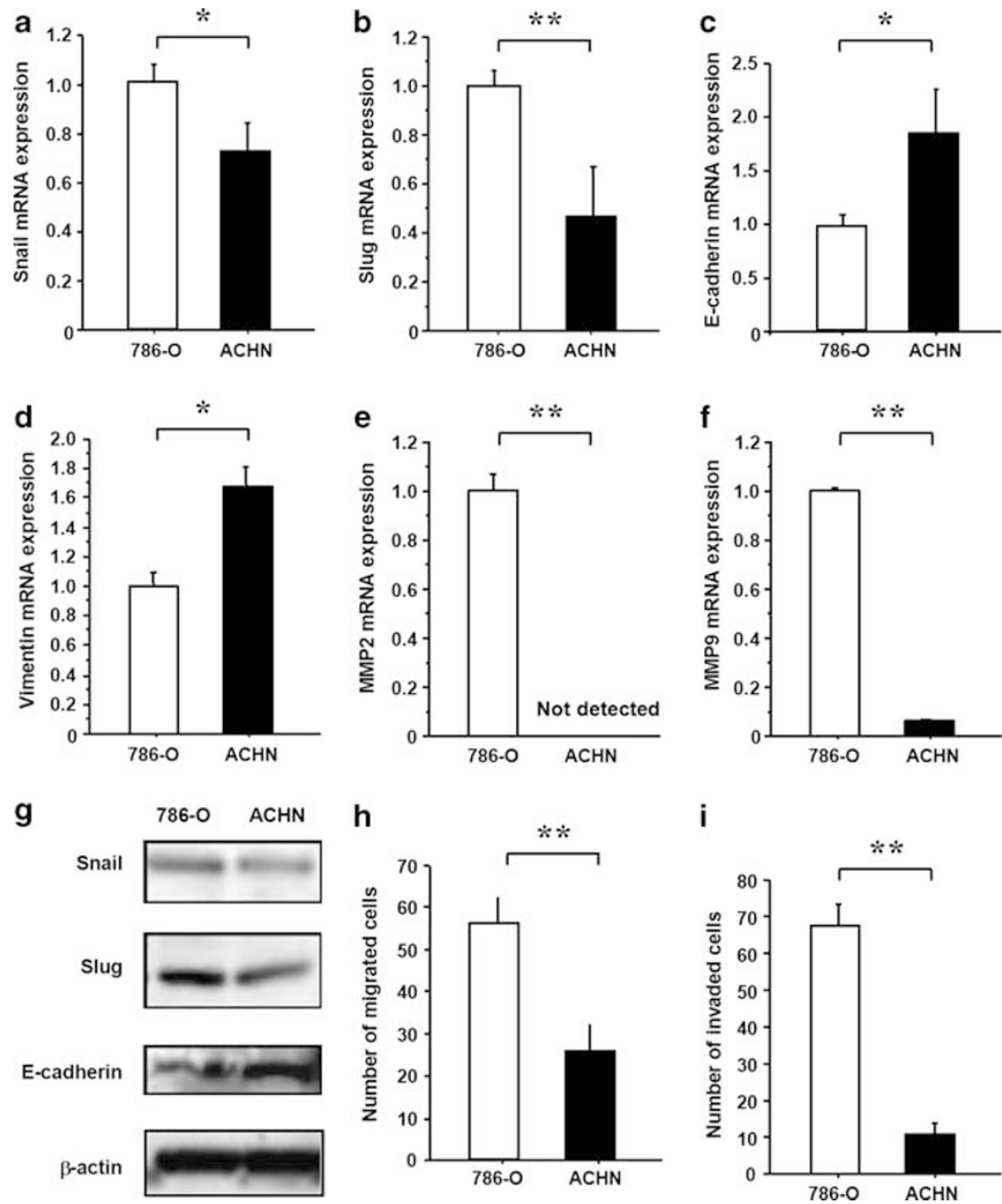

Figure 4 Expression of Snail (a), Slug (b), E-cadherin (c), vimentin (d), MMP2 (e) and MMP9 (f) mRNA in 786-O and ACHN cells. Immunoblot analysis of Snail, Slug and E-cadherin (g). The migrated cells (h) and invaded cells (i) were quantified as described in Materials and methods. Columns, means; bars, s.d.; ${ }^{*} P<0.05 ;{ }^{*} P<0.01$. 
than in ACHN cells (Figures 4e and f). Snail, Slug and E-cadherin expression was investigated by immunoblot analysis (Figure $4 \mathrm{~g}$ ). The number of migrated cells was twofold higher in 786-O cells than ACHN cells (Figure 4h), and the Matrigel invasion assay indicated that $786-\mathrm{O}$ cells showed more invasive activity than ACHN cells (Figure 4i). Because 786-O cells showed the higher Snail expression, more migration and invasive activity, they were used for transfection with siRNA for Snail as described below.

\section{Effect of siRNA for Snail on Matrigel Invasion by RCC Cells In Vitro}

Transfection of two different types of siRNA for Snail (siSnail\#1 and siSnail\#2) in 786-O cells reduced the mRNA expression levels of Snail by 45 and $42 \%(P<0.05)$ compared with that of the cells transfected with NSi (Figure 5a). siRNAs for Snail did not affect the Slug mRNA expression level (Figure 5b). The expression level of E-cadherin mRNA increased by 23 or $40 \%$ in the cells transfected with siSnail\# 1 or siSnail\#2, respectively, compared with that of the NSitransfected cells $(P<0.05$; Figure $5 \mathrm{c})$. The cells transfected with siSnail\#1 or siSnail\#2 showed decreased expression of vimentin mRNA by 52 or $19 \%$, respectively, compared with that of those with NSi $(P<0.05$; Figure $5 \mathrm{~d})$. The cells transfected with siSnail\#1 or siSnail\#2 showed decreased expression of MMP2 mRNA by 46 or $32 \%$, respectively, compared with the cells transfected with NSi $(P<0.05$; Figure 5e). Similarly, decreased expression of MMP9 mRNA (56 and 62\%) was observed by the transfection with siSnail\#1 or siSnail\#2 $(P<0.01$; Figure 5f). However, transfection of siSnail\#1 or siSnail\#2 showed no significant effect on the expression of MMP14 (data not shown). Immunoblot analysis also revealed that decreased Snail expression and increased E-cadherin expression in the cell transected with siRNAs for Snail (Figure 5g).

The number of migrated cells was significantly lower in 786-O cells transfected with siSnail\#1 or siSnail\#2 (30.7 \pm 2.1 or $32.0 \pm 4.4)$ than those transfected with NSi $(53.7 \pm 4.7)$ $(P<0.01$; Figure 5 h). $786-\mathrm{O}$ cells transfected with siSnail\#1
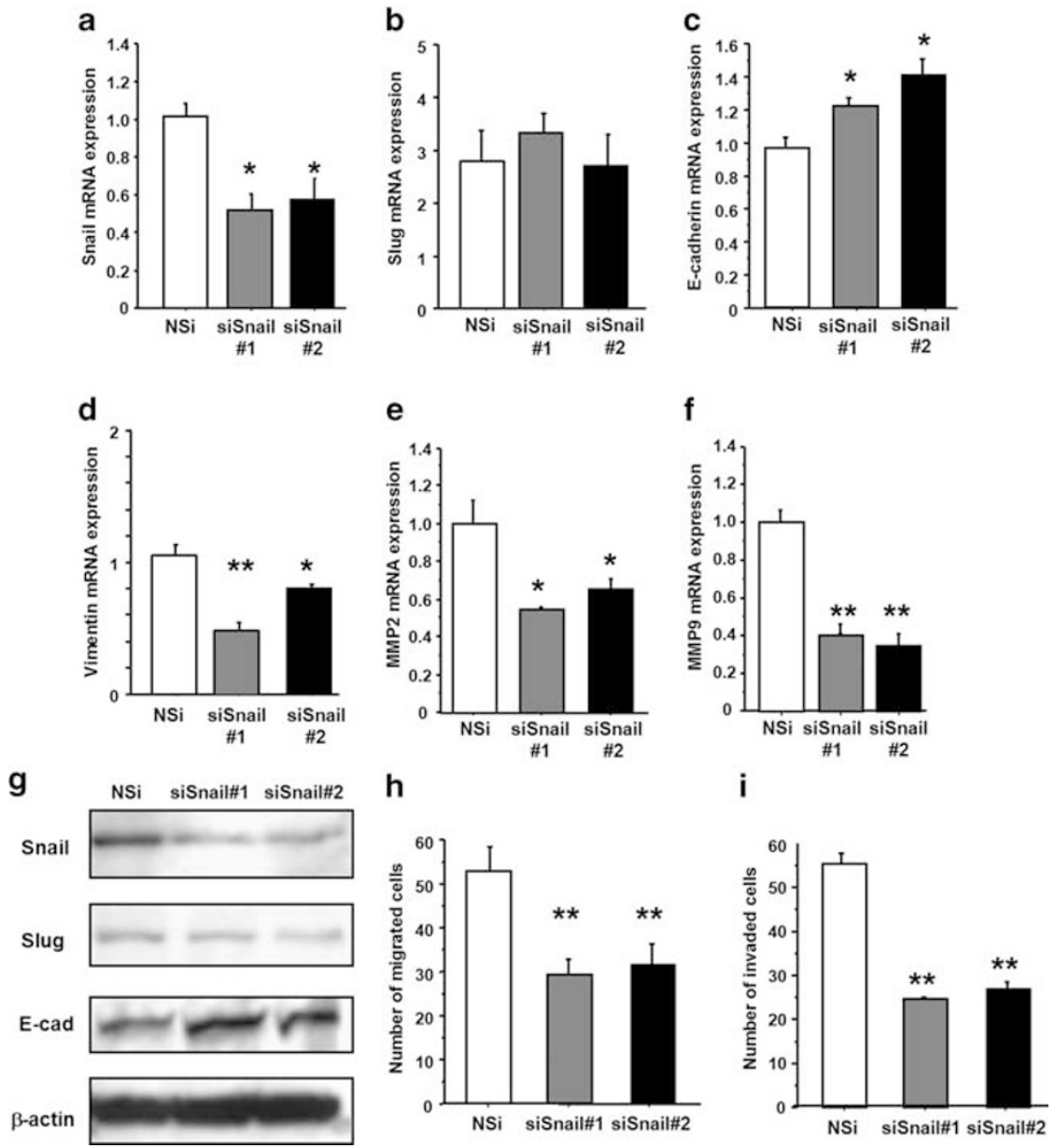

Figure 5 Expression of Snail (a), Slug (b), E-cadherin (c), vimentin (d), MMP2 (e), MMP9 (f) mRNA in 786-O cells with or without targeting Snail (siSnail\#1, siSnail\#2) and cells with NSi. Immunoblot analysis of Snail, Slug and E-cadherin (g). The migrated cells (h) and invaded cells (i) were quantified as described in Materials and methods. Columns, mean; bars, s.d.; ${ }^{\star} P<0.05 ;{ }^{*} P<0.01$. 
or siSnail\#2 exhibited decreased invasion activity $(24.3 \pm 1.2$ or $27.3 \pm 2.5)$ compared with those transfected with NSi $(54.7 \pm 5.5)(P<0.01$; Figure 5i).

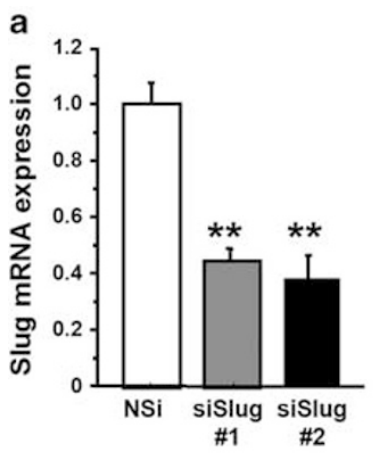

b
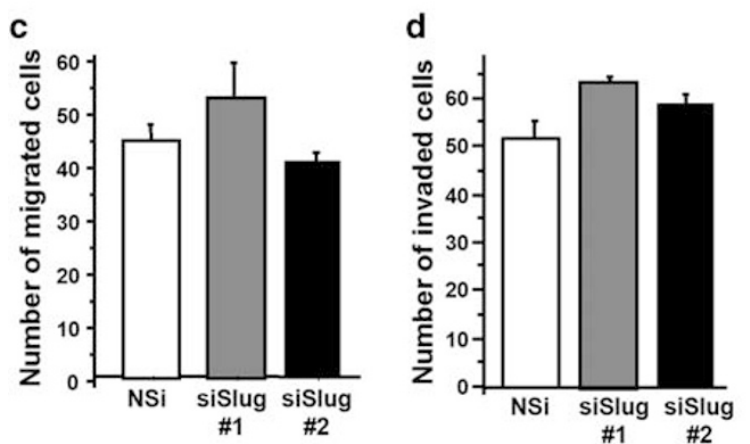

Figure 6 Expression of Slug mRNA (a), protein (b), migration (c) and invasion (d) in 786-O cells with targeting Slug (siSlug\#1 and siSlug\#2) and cells with NSi.
Effect of siRNA for Slug on Invasion by RCC Cells In Vitro siRNAs for Slug (siSlug\#1 and siSlug\#2) reduced the mRNA expression of Slug by 52 and $58 \%(P<0.01)$ compared with the cells transfected with NSi (Figure 6a). Slug protein expression was also decreased by siRNAs for Slug (Figure 6b). However, siRNA for Slug did not affect the expression level of Snail, E-cadherin, MMP2 or MMP9 mRNA (data not shown). In addition, no significant difference of cancer cell migration and invasion was observed between the cells transfected with siRNAs for Slug or NSi (Figures $6 c$ and d).

\section{Expression of MMP2 and MMP9 in RCCs and Non-Neoplastic Renal Tissues}

Because in vitro studies described above revealed that Snail regulates MMP2 and MMP9, these MMPs expression in RCCs were investigated. Immunoblot analysis demonstrated that MMP2 protein is expressed in one of four low-grade clear cell RCCs and all three high-grade clear cell RCCs (Figure 1f). Immunohistochemically, clear cell RCCs with G2 showed focal MMP2 protein expression (Figure 7a), and many cancer cells of clear cell RCC with G3 were positive for MMP2 staining (Figure 7b). Furthermore, sarcomatoid carcinoma showed diffuse and strong MMP2 expression (Figure 7c). The mean MMP2-positive cancer cell ratio was $44 \pm 3$ in clear cell RCCs, and it was similar to those in papillary $(44 \pm 28)$ and chromophobe RCCs $(44 \pm 33)$. MMP2-positive cancer cell ratio was correlated with pathological tumor stage $(P<0.001)$, distant metastasis $(P=0.016)$, histological grade $(P<0.001)$, venous invasion $(P=0.003)$ and the presence of sarcomatoid carcinoma $(P=0.013)$ (Table 2).
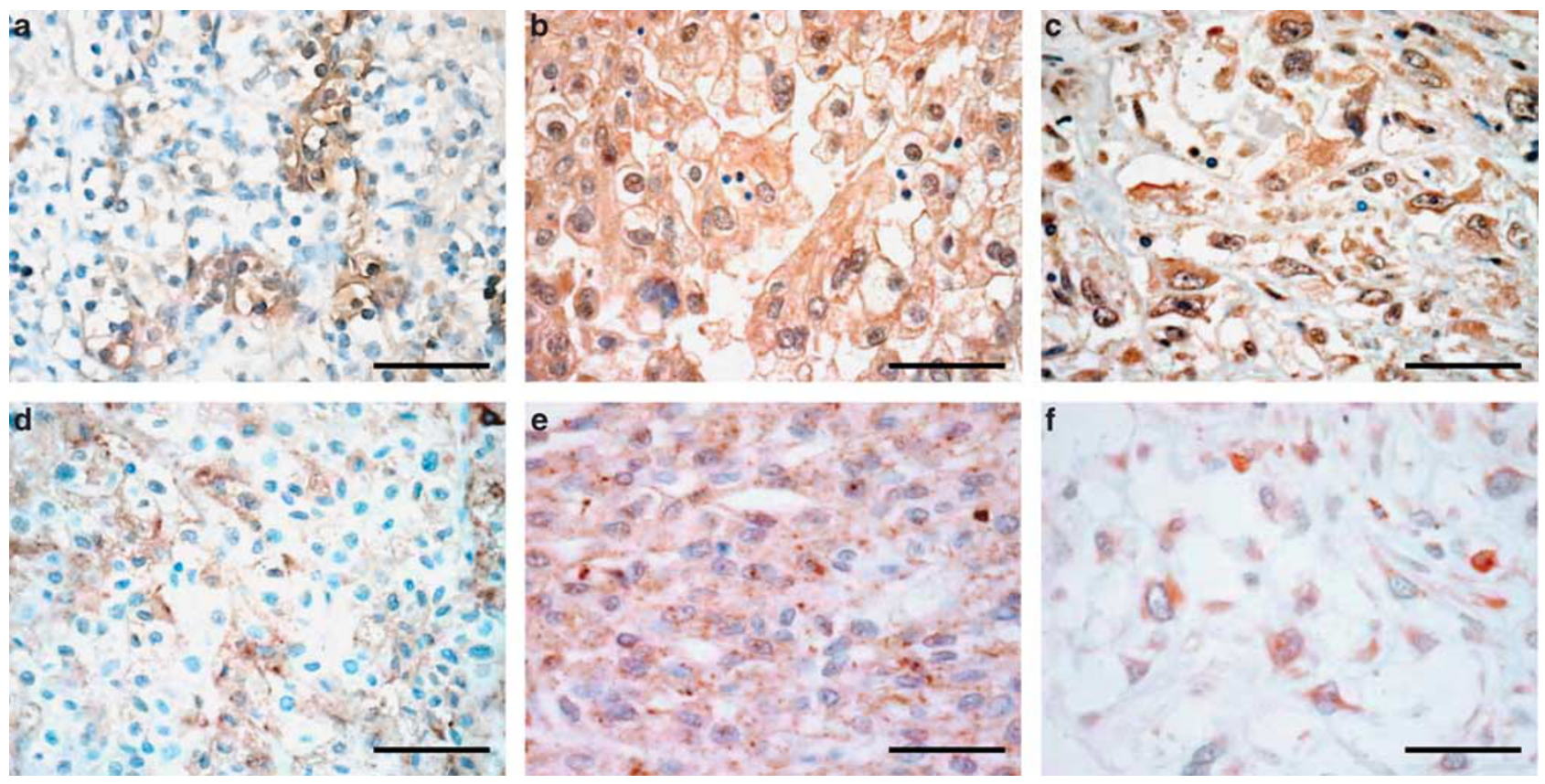

Figure 7 Immunostaining of MMP2 in clear cell RCC, G2 (a), clear cell RCC, G3 (b), sarcomatoid carcinoma (c). Immunostaining of MMP9 in clear cell RCC, G2 (d), clear cell RCC, G3 (e) and sarcomatoid carcinoma (f). Bars, $50 \mu \mathrm{m}$. 
Table 2 Relationship between expression of MMP2, MMP9 and clinicopathological parameters in renal cell carcinomas

\section{MMP2-positive tumor MMP9-positive tumor cells (\%) median cells (\%) median}

(25th-75th percentile) (25th-75th percentile)

$$
\begin{aligned}
& \text { Gender } \\
& \text { Male }(n=74) \\
& \text { Female }(n=23) \\
& P
\end{aligned}
$$

Age (years)

Median or less $(\leq 59)(n=48)$

Over median (>59) $(n=49)$

$P$

Pathological tumor stage

pT1, 2 ( $n=78)$

pT3, $4(n=19)$

$P$

\section{Lymph node metastasis \\ Negative $(n=93)$ \\ Positive $(n=4)$ \\ $P$}

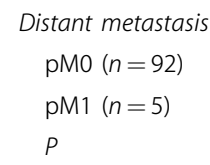

$$
\begin{gathered}
31.5(17.5-70.0) \\
50.0(23.8-71.3) \\
0.228
\end{gathered}
$$

$$
\begin{aligned}
& 31.0(15.0-67.0) \\
& 77.0(56.3-85.0)
\end{aligned}
$$

$<0.001$

\section{$35.0(20.0-70.0)$ \\ $67.5(39.0-75.0)$ \\ 0.457}

$33.0(10.0-58.3)$

$63.5(33.5-72.5)$

0.217

$34.0(15.0-61.0)$
$33.0(10.0-62.8)$
0.817

$27.5(10.0-45.0)$

$70.0(45.0-80.0)$

$<0.001$

0.879

.

$33.0(11.0-55.0)$

$72.0(53.8-88.3)$

0.043

$$
\begin{gathered}
35.0(20.0-70.0) \\
80.0(68.8-85.0) \\
0.016
\end{gathered}
$$

$30.0(15.0-65.0)$
$76.0(57.0-87.3)$
$<0.001$

$27.0(10.0-45.0)$

$66.0(29.8-75.8)$

$<0.001$

$$
\begin{aligned}
& \text { Venous invasion } \\
& \text { Negative }(n=74) \\
& \text { Positive }(n=23) \\
& P
\end{aligned}
$$
Sarcomatoid carcinoma
Negative $(n=90)$
Positive $(n=7)$
P

By immunoblot analysis, MMP9 protein expression was observed in one of four low-grade clear cell RCCs and two of three high-grade clear cell RCCs. Clear cell RCCs with G2 showed focal MMP9 protein expression (Figure 7d), and many cancer cells of clear cell RCCs with G3 were positive for MMP9 staining (Figure 7e). Furthermore, sarcomatoid carcinoma showed diffuse and strong MMP9 expression (Figure 7f). The mean MMP9-positive cancer cell ratio was $38 \pm 28$ in clear cell RCCs, and it was similar to those in papillary $(28 \pm 28)$ and chromophobe RCCs $(34 \pm 31)$. MMP9-positive cancer cell ratio was correlated with pathological tumor stage $(P<0.001)$, distant metastasis $(P=0.043)$, histological grade $(P<0.001)$ and the presence of sarcomatoid carcinoma $(P=0.021)$ (Table 2$)$.

\section{Relationships Between Expression of Snail, Slug, E-cadherin and Clinicopathological Parameters in Clear Cell RCCs}

As shown in Figure 3, the expression of Snail, Slug and E-cadherin in clear cell RCCs was different from that in papillary and chromophobe RCCs. However, the relationships between Snail, Slug, E-cadherin, MMP2 and MMP9 and clinicopathological parameters in 83 clear cell RCCs were essentially similar to those in 97 RCCs including all subtypes of RCCs (Tables 1-4).

\section{Correlations Between Expression of Snail, Slug, E-cadherin, MMP2 and MMP9 in RCCs and Survival of the Patients}

Patients with Snail-low tumors exhibited longer disease-free survival than those with Snail-high tumors $(P=0.0010$; Figure 8a). Patients with Snail-low tumors exhibited significantly longer disease-specific survival than those with Snailhigh tumors $(P=0.0149$; Figure $8 \mathrm{~b})$.

Patients with Slug-low tumors exhibited shorter diseasefree survival than those with Slug-high tumors $(P=0.0402$; Figure 8c). Patients with Slug-low tumors exhibited significantly shorter disease-specific survival than those with Slug-high tumors $(P=0.0417$; Figure 8d).

Patients with E-cadherin-high tumors exhibited slightly longer disease-free survival than those with E-cadherin-low tumors, but the difference did not reach statistically significance $(P=0.0833$; Figure $8 \mathrm{e})$. However, no significant association was found between E-cadherin expression and disease-specific survival ( $P=0.3276$; Figure $8 \mathrm{f}$ ).

Patients with MMP2-low tumors exhibited longer diseasefree survival than those with MMP2-high tumors $(P=0.0003$; Figure 8g). Patients with MMP2-low tumors exhibited significantly longer disease-specific survival than those with MMP2-high tumors ( $P=0.0249$; Figure $8 \mathrm{~h}$ ).

Patients with MMP9-low tumors exhibited longer diseasefree survival than those with MMP9-high tumors $(P=0.0097$; Figure 8i). Patients with MMP9-low tumors exhibited significantly longer disease-specific survival than those with MMP9-high tumors $(P=0.0267$; Figure 8j).

Pathological tumor stage, distant metastasis, histological grade, venous invasion, sarcomatoid carcinoma and Snail, Slug, MMP2, MMP9 expression were the prognostic factors of disease-free and disease-specific survival by univariate analysis (data not shown). By multivariate analysis, however, 
Table 3 Relationship between expression of Snail, Slug, E-cadherin and clinicopathological parameters in clear cell renal cell carcinomas

Snail-positive tumor

cells (\%) median

(25th-75th percentile)

Gender

Male $(n=62)$

Female $(n=21)$

$P$

Age (years)

Median or less $(\leq 59)(n=39)$

$25.0(11.3-47.3)$

$2.0(0-29.5)$

Over median (>59) $(n=44)$

P

$25.0(7.0-69.0)$

0.895

$5.5(0-29.0)$

0.750

$0(0-15.0)$

$20.0(0-53.0)$

0.003

Pathological tumor stage

pT1, $2(n=65)$

pT3, $4(n=18)$

$P$

$24.0(6.5-45.0)$

$66.0(23.0-77.0)$

0.016

\author{
$25.0(8.0-60.0)$ \\ $65.0(1.5-70.0)$ \\ 0.616
}

$P$

Distant metastasis

$$
\text { pMO }(n=79)
$$

pM1 $(n=4)$

$P$

$25.0(7.3-62.5)$
$54.5(35.0-78.0)$
0.092

Histological grade

$$
\begin{aligned}
& \mathrm{G} 1,2(n=64) \\
& \mathrm{G} 3,4(n=19)
\end{aligned}
$$

$P$

\section{Venous invasion}

Negative $(n=64)$

Positive $(n=19)$

P

Sarcomatoid carcinoma

Negative $(n=76)$

Positive $(n=7)$

P

\author{
23.5 (4.0-41.5) \\ $55.0(25.0-77.0)$ \\ 0.009
}
$24.0(6.0-45.0)$
$55.0(18.0-77.0)$
0.031
$24.5(7.0-51.5)$
$71.0(50.5-83.0)$
0.010

$10.0(0-30.0)$
$1.0(0-3.0)$
0.038

$2.0(0-35.0)$

$16.0(0-23.0)$

0.855
$6.5(0-30.0)$

$23.0(5.8-32.0)$

0.696

$10.0(0-32.5)$

$12.5(1.0-37.0)$

0.695

$\begin{array}{cc}5.0(0-30.0) & 10.0(0-32.5) \\ 1.5(0.5-8.5) & 12.5(1.0-37.0) \\ 0.449 & 0.695\end{array}$

$2.5(0-25.0)$

$2.0(0-12.3)$

0.229

$20.0(0-35.0)$

0.481
$9.0(0-35.0)$
$10.0(0-24.5)$
0.828
$5.0(0-30.0)$
$2.0(0.3-22.5)$
0.676

$6.5(0-30.0)$
$11.0(0-35.0)$
$0(0-22.8)$

0.577
E-cadherin-positive tumor cells (\%) median (25th-75th percentile) 
Table 4 Relationship between expression of MMP2, MMP9 and clinicopathological parameters in clear cell renal cell carcinomas

MMP2-positive tumor MMP9-positive tumor cells (\%) median cells (\%) median

(25th-75th percentile) (25th-75th percentile)

\section{Gender}

Male $(n=62)$

Female $(n=21)$

$P$

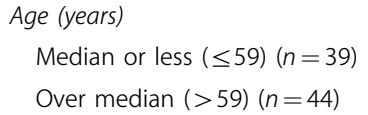
Sarcomatoid carcinoma
Negative $(n=76)$
Positive $(n=7)$
P

$$
\begin{array}{cc}
47.5(20.0-70.0) & 33.5(15.0-57.0) \\
30.0(19.8-56.3) & 35.0(5.0-71.3) \\
0.168 & 0.785
\end{array}
$$

30.0 (16.3-69.8) $52.5(22.5-72.5)$

0.292

$33.0(15.5-54.5)$ 34.5 (12.5-67.5) 0.482

$$
\begin{array}{cc}
30.0(15.0-67.0) & 31.3(11.5-42.8) \\
78.5(55.0-85.0) & 71.5(45.0-80.0) \\
<0.001 & <0.001
\end{array}
$$

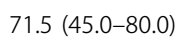

$$
\begin{gathered}
35.0(20.0-70.0) \\
65.0(26.0-76.3) \\
0.733
\end{gathered}
$$

33.5 (11.5-63.5) $55.0(22.8-68.5)$ 0.550

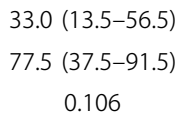

0.027

$$
\begin{aligned}
& 30.0(14.5-66.0) \\
& 77.0(55.0-87.3)
\end{aligned}
$$$$
<0.001
$$

$$
\begin{aligned}
& 27.0(8.5-45.0) \\
& 68.0(45.8-79.0)
\end{aligned}
$$$$
<0.001
$$$$
\begin{gathered}
33.0(12.0-55.0) \\
53.0(25.0-72.3) \\
0.108
\end{gathered}
$$

$30.0(15.0-67.0)$ 70.0 (42.5-85.0) 0.002 0.108

\section{$33.0(12.5-55.0)$ $70.0(48.0-82.7)$ 0.024 \\ 33.5 (17.5-69.5) $85.0(70.3-88.0)$ 0.013}

\section{DISCUSSION}

In the present study, we have provided the evidence that Snail is immunolocalized to carcinoma cells in high-grade RCCs with correlations to pathological tumor stage and the presence of sarcomatoid carcinoma, and that all metastatic RCCs show strong Snail expression with decreased E-cadherin expression. These data suggest that overexpression of Snail is associated with tumor aggressiveness of RCCs by inducing EMT. Furthermore, elevated Snail expression was identified as a worse prognostic factor for disease-free and diseasespecific survival in RCC patients. These findings are in agreement with the data reported by previous studies showing positive correlations of Snail expression with enhanced metastatic spread and reduced post-operative survival from human cancers such as breast, endometrial and ovarian cancers. ${ }^{15-19}$

Sarcomatoid carcinoma represents high-grade transformation of RCCs, and RCCs with sarcomatoid carcinoma tend to develop more advanced stages. ${ }^{20}$ Because morphological transformation from low-grade RCCs into high-grade RCCs, and especially sarcomatoid carcinomas, appears to represent EMT of RCCs, we investigated the expression of Snail, a key transcriptional factor of EMT, and E-cadherin in RCCs. All sarcomatoid carcinomas showed strong Snail expression without E-cadherin expression. This reverse correlation of the expression of Snail and E-cadherin was experimentally confirmed in the RCC cells by transfection of siRNA for Snail. These findings suggest that Snail is involved in EMT of RCCs, leading to a morphological phenotype of sarcomatoid carcinomas.

By real-time PCR analysis, E-cadherin mRNA expression was higher in non-neoplastic renal tissues than that in clear cell RCCs, but no significant difference was observed between Snail expression in non-neoplastic renal tissues and that in clear cell RCCs. This is probably due to some neoplastic renal tissues that showed relatively high Snail mRNA expression. The non-neoplastic renal tissues investigated in the present study involve not only renal tubules and glomeruli but also mesenchymal cells, such as fibroblasts and inflammatory cells. Immunohistologically, Snail staining was observed in fibroblasts and inflammatory cells in non-neoplastic renal tissue of some cases. Similarly, Snail-positive stromal cells have been described in ovarian carcinomas and in endometrial and colon carcinomas. ${ }^{16,17}$ This may be the reason why non-neoplastic renal tissues in some cases showed relatively high Snail mRNA expression. Although Snailpositive stromal cells may mediate the mesenchymal phenotype of the cells, the meaning of Snail expression in tumor-associated stromal cells remains unclear.

Previous in vitro studies have suggested the possibility that VHL may have a role in EMT of RCCs and that Snail may be involved in the process. ${ }^{11-13}$ Because it is well known that VHL loss of function is an early event of clear cell RCCs, EMT induced by Snail is expected to occur in early stage clear cell RCCs. However, early stage clear cell RCCs show

no independent prognostic factor was found (data not shown).

When correlation studies were performed in just 83 clear cell RCCs, essentially the similar results were observed compared with those obtained with all subtypes of RCCs (data not shown). 

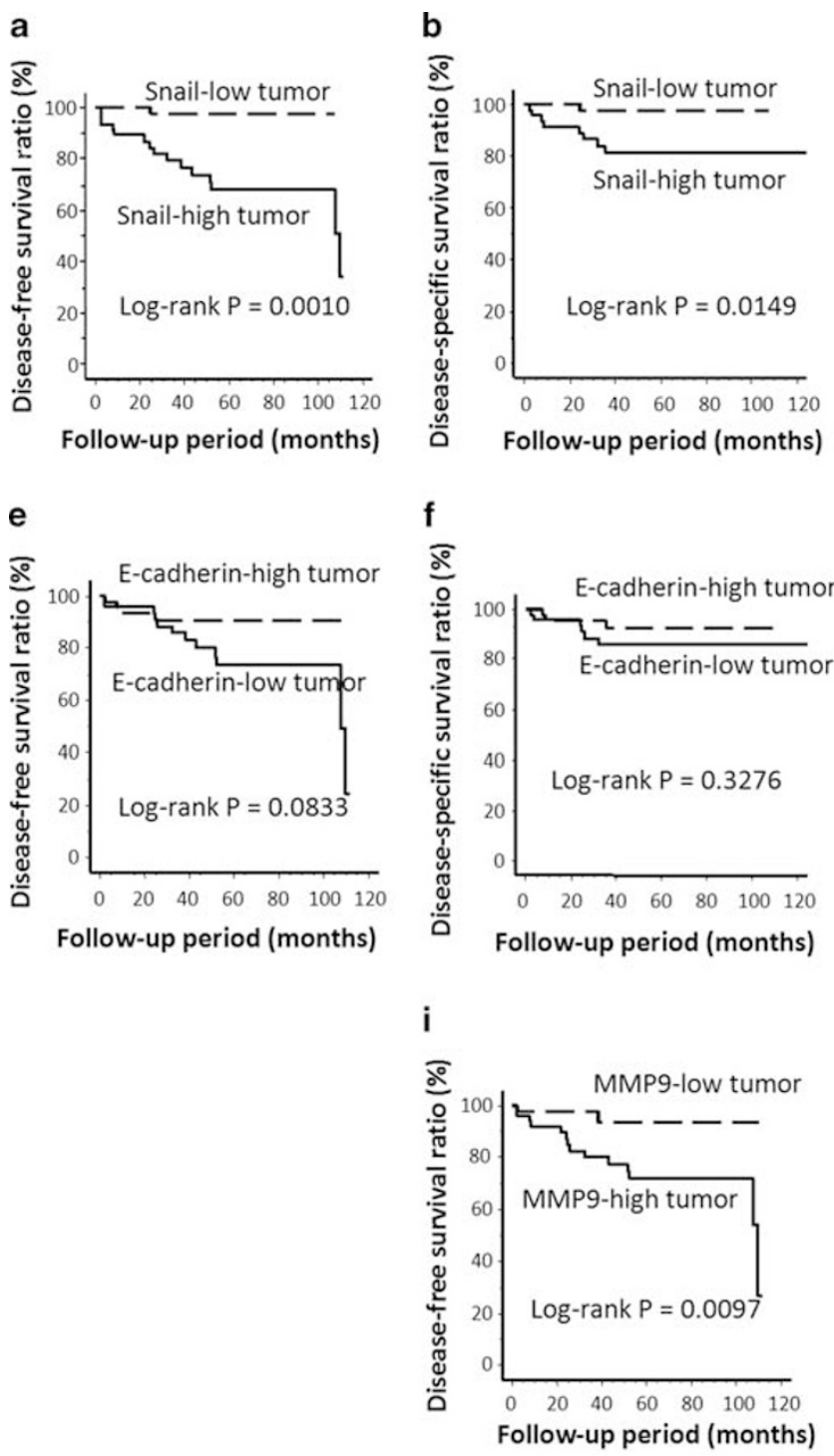
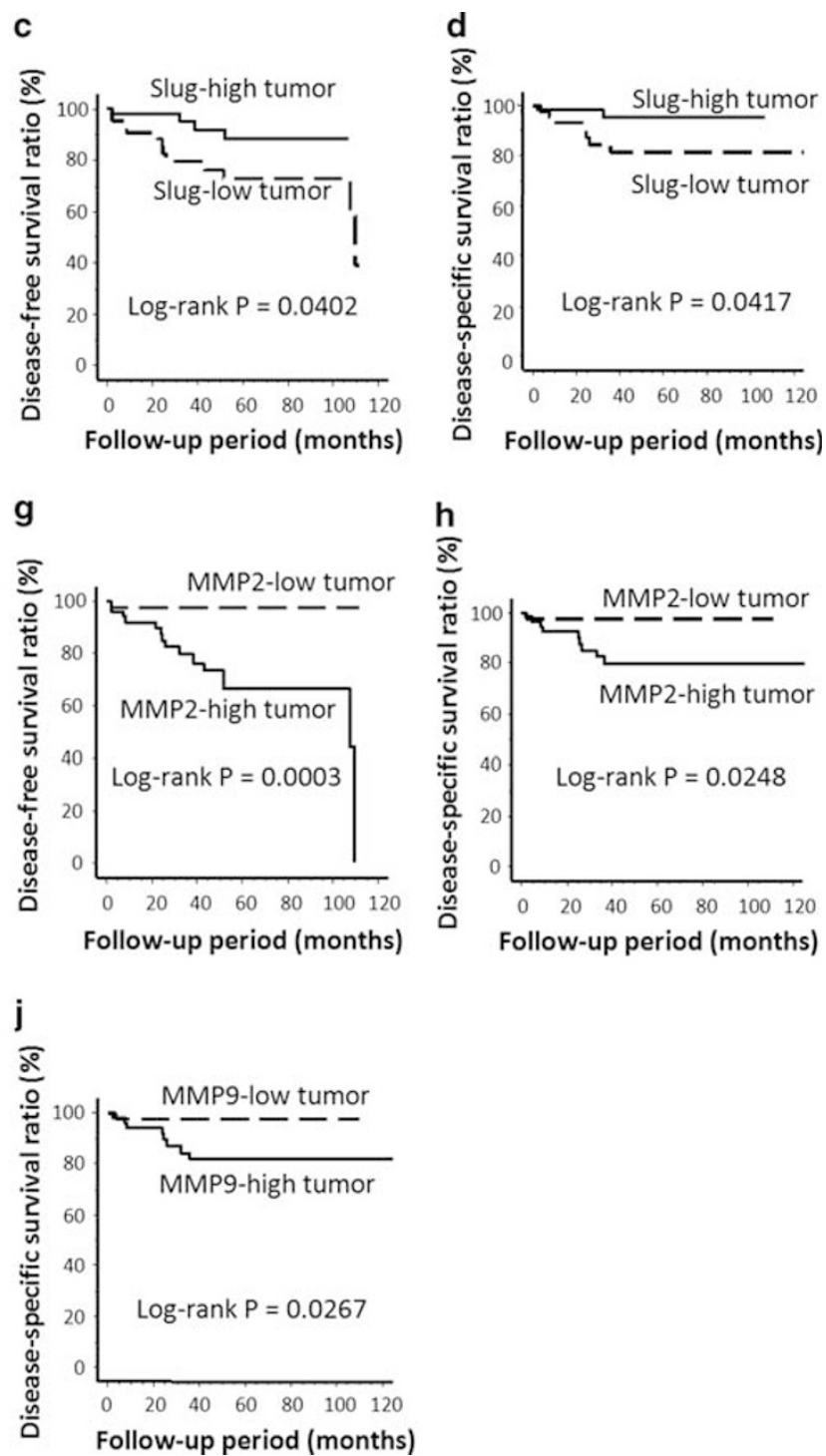

Figure 8 Kaplan-Meier curves of disease-free survival and disease-specific survival according to Snail (a, b), Slug (c, d), E-cadherin (e, f), MMP2 (g, h) and MMP9 (i, j) expression in 97 patients with RCC including all subtypes of RCCs.

epithelial morphology with preserved E-cadherin expression. ${ }^{10}$ Messai et al $^{21}$ reported that Snail expression is lower in low-grade RCCs than in high-grade RCCs, suggesting that up-regulation of Snail may be important in EMT of RCCs in vivo. However, Messai et al ${ }^{21}$ examined the expression of Snail to investigate its correlation with cytokeratin 18 expression, and they did not investigate the role of Snail expression in association with EMT of RCCs. Accordingly, the role of Snail in EMT and its association with cancer invasion, metastasis and prognosis of RCCs has not been established yet.

Slug is expressed in several carcinomas with increased invasion through regulation of E-cadherin. ${ }^{9}$ In the present study, however, Slug mRNA expression was lower in RCCs than that in non-neoplastic renal tissues. Slug protein expression was lower in pT3-4 RCCs compared with pT1-2
RCCs. These indicate that Slug is rather down-regulated during carcinogenesis and malignant progression of RCCs, suggesting that Slug may not have a role in the EMT of RCCs. Our data of the experiment using siRNA for Slug demonstrated no effects on cancer cell migration or invasion in vitro. Although Snail is known to regulate E-cadherin expression in mouse keratinocytes, Slug appears to have insignificant role in this process. ${ }^{22}$ It is also reported that Slug is neither a repressor of E-cadherin nor an inducer of cell movement during EMT of renal tubulogenesis but that Slug is required for cell survival. ${ }^{23}$ Although many studies have shown that Snail and Slug are positive regulators of EMT, ${ }^{24}$ we propose the hypothesis that Snail is a positive regulator of EMT in RCCs and Slug has other roles in RCCs, such as cell survival. 
It was reported that von Hippel-Lindau gene (VHL) is mutated in 786-O cells, and it was wild type in ACHN cells. ${ }^{25}$ In the present study, 786-O cell showed relatively high Snail expression and low E-cadherin expression compared with ACHN cells. It may be related to that VHL mutation induce hypoxia inducible factor-1 stabilization and this may repress E-cadherin expression via induction of Snail. ${ }^{12}$ Because the difference in invasion of the two cell lines cannot be fully explained by E-cadherin expression alone, we analyzed MMP2 and MMP9 expression in these cell lines. As a result, both MMPs expression was markedly higher in 786-O cells than that in ACHN cells, suggesting that MMPs may be related to the invasion of these cell lines.

In the present study, down-regulation of Snail by siRNA resulted in decreased cancer cell migration, and it was probably because the cell-cell adhesion was restored by upregulated E-cadherin expression. Furthermore, knockdown of Snail expression inhibited cancer cell invasion through Matrigel, and this effect cannot be fully explained by only upregulation of E-cadherin expression, because the ability of cancer cells to invade extracellular matrix and basement membrane and establish distant metastasis requires the disruption of tissue barriers and the modulation of cell-cell and cell-matrix contacts. ${ }^{26}$ Members of the MMP family, in particular MMP2 and MMP9, have an important role in cell migration and invasion, ${ }^{27}$ and overexpression of Snail increases the expression of MMP9 in MDCK cells. ${ }^{28}$ It is also reported that knockdown of Snail expression suppresses the activity of MMP2 and MMP9 in ovarian cancer cells in vitro. ${ }^{19}$ In the present study, siRNA for Snail suppressed expression of MMP2 and MMP9 and invasion through Matrigel in vitro. These findings indicate that Snail has an essential role in regulating the expression of MMPs during invasion and metastasis. In fact, expression level of MMPs was associated with many clinicopathological parameters, and high expression of MMPs was predictors of disease-free and disease-specific survivals.

In conclusion, we have shown the predominance of the expression of Snail in high-grade RCCs compared with that in low-grade RCCs. High-Snail expression was found to be associated with malignant potential of RCCs, and it was correlated with poor prognosis. Furthermore, we have provided the data that down-regulation of Snail, but not Slug, expression effectively inhibits the invasion of RCC cells through Matrigel in vitro together with up-regulation of E-cadherin and down-regulation of MMP2 and MMP9. These data suggest that Snail has a substantial role in EMT and the malignant phenotype of RCC not only by loss of cell-cell adhesion but also by penetration of basement membrane structure. Accordingly, targeting of Snail expression may be an anti-tumor therapy for RCCs.

\section{ACKNOWLEDGEMENTS}

This work was supported in part by Grant-in-Aid for Young Scientists (B) (No. 21790365) (SM), Grain-in-Aid for Scientific Research (B) (No. 21390445) (MO), and Grant-in-Aid for Scientific Research (S) (No. 19109004) (YO) from the Ministry of Education, Culture, Sports, Science, and Technology of Japan.

\section{DISCLOSURE/CONFLICT OF INTEREST}

The authors declare no conflict of interest.

1. Eble JN, Saunders G, Epstein Jl, et al. (eds). World Health Organization Classification of Tumors. Pathology and Genetics of Tumors of the Urinary System and Male Genital Organs. IARC Press: Lyon, 2005.

2. Zisman A, Pantuck AJ, Wieder J, et al. Risk group assessment and clinical outcome algorithm to predict the natural history of patients with surgically resected renal cell carcinoma. J Clin Oncol 2002;20:4559-4566.

3. Engbring JA, Kleinman HK. The basement membrane matrix in malignancy. J Pathol 2003;200:465-470.

4. Chambers AF, Matrisian LM. Changing views of the role of matrix metalloproteinases in metastasis. J Natl Cancer Inst 1997;89: $1260-1270$

5. Kugler A, Hemmerlein B, Thelen $\mathrm{P}$, et al. Expression of metalloproteinase 2 and 9 and their inhibitors in renal cell carcinoma. J Urol 1998;160:1914-1918.

6. Grunert $S$, Jechlinger $M$, Beug $H$. Diverse cellular and molecular mechanisms contribute to epithelial plasticity and metastasis. Nat Rev Mol Cell Biol 2003;4:657-665.

7. Thiery JP, Sleeman JP. Complex networks orchestrate epithelialmesenchymal transitions. Nat Rev Mol Cell Biol 2006;7:131-142.

8. Haraguchi M. The role of the transcriptional regulator snail in cell detachment, reattachment and migration. Cell Adh Migr 2009;3: 259-263.

9. Alves CC, Carneiro F, Hoefler $\mathrm{H}$, et al. Role of the epithelialmesenchymal transition regulator Slug in primary human cancers. Front Biosci 2009;14:3035-3050.

10. Katagiri A, Watanabe R, Tomita Y. E-cadherin expression in renal cell cancer and its significance in metastasis and survival. $\mathrm{Br} J$ Cancer 1995;71:376-379.

11. Esteban MA, Tran MG, Harten SK, et al. Regulation of E-cadherin expression by $\mathrm{VHL}$ and hypoxia-inducible factor. Cancer Res 2006;66:3567-3575.

12. Evans AJ, Russell RC, Roche $\mathrm{O}$, et al. VHL promotes E2 box-dependent E-cadherin transcription by HIF-mediated regulation of SIP1 and snail. Mol Cell Biol 2007;27:157-169.

13. Harten SK, Shukla D, Barod R, et al. Regulation of renal epithelial tight junctions by the von Hippel-Lindau tumor suppressor gene involves occludin and claudin 1 and is independent of E-cadherin. Mol Biol Cell 2009;20:1089-1101.

14. Mikami S, Oya M, Shimoda M, et al. Expression of heparanase in renal cell carcinomas: implications for tumor invasion and prognosis. Clin Cancer Res 2008;14:6055-6061.

15. Blanco MJ, Moreno-Bueno G, Sarrio D, et al. Correlation of Snail expression with histological grade and lymph node status in breast carcinomas. Oncogene 2002;21:3241-3246.

16. Blechschmidt K, Kremmer E, Hollweck R, et al. The E-cadherin repressor snail plays a role in tumor progression of endometrioid adenocarcinomas. Diagn Mol Pathol 2007;16:222-228.

17. Franci C, Takkunen M, Dave N, et al. Expression of Snail protein in tumor-stroma interface. Oncogene 2006;25:5134-5144.

18. Jiao W, Miyazaki K, Kitajima Y. Inverse correlation between E-cadherin and Snail expression in hepatocellular carcinoma cell lines in vitro and in vivo. Br J Cancer 2002;86:98-101.

19. Jin $\mathrm{H}, \mathrm{Yu} \mathrm{Y}, \mathrm{Zhang} \mathrm{T}$, et al. Snail is critical for tumor growth and metastasis of ovarian carcinoma. Int J Cancer 2010;126:2102-2111.

20. Delahunt B. Sarcomatoid renal carcinoma: the final common dedifferentiation pathway of renal epithelial malignancies. Pathology 1999;31:185-190.

21. Messai $Y$, Noman $M Z$, Derouiche $A$, et al. Cytokeratin 18 expression pattern correlates with renal cell carcinoma progression: relationship with Snail. Int J Oncol 2010;36:1145-1154.

22. Cano A, Perez-Moreno MA, Rodrigo I, et al. The transcription factor snail controls epithelial-mesenchymal transitions by repressing E-cadherin expression. Nat Cell Biol 2000;2:76-83. 
23. Leroy $\mathrm{P}$, Mostov KE. Slug is required for cell survival during partial epithelial-mesenchymal transition of HGF-induced tubulogenesis. Mol Biol Cell 2007;18:1943-1952.

24. Nieto MA. The snail superfamily of zinc-finger transcription factors. Nat Rev Mol Cell Biol 2002;3:155-166.

25. Shinojima T, Oya M, Takayanagi $A$, et al. Renal cancer cells lacking hypoxia inducible factor (HIF)-1alpha expression maintain vascular endothelial growth factor expression through HIF-2alpha. Carcinogenesis 2007;28:529-536.
26. Roy R, Yang J, Moses MA. Matrix metalloproteinases as novel biomarkers and potential therapeutic targets in human cancer. J Clin Oncol 2009;27:5287-5297.

27. Orlichenko LS, Radisky DC. Matrix metalloproteinases stimulate epithelial-mesenchymal transition during tumor development. Clin Exp Metastasis 2008;25:593-600.

28. Jorda M, Olmeda D, Vinyals A, et al. Upregulation of MMP-9 in MDCK epithelial cell line in response to expression of the Snail transcription factor. J Cell Sci 2005;118:3371-3385. 